\title{
Electrical Stimulation of the Amygdala Modifies the Negative Feedback Effect of Glucocorticoids on the Adrenocortical Responses to Stress
}

\author{
Joseph Weidenfeld Anna Itzik Haim Ovadia \\ Department of Neurology, The Agnes Ginges Center for Human Neurogenetics, Hadassah-Hebrew University \\ Medical Center, Jerusalem, Israel
}

\section{Key Words}

Amygdala - Electrical stimulation .

Hypothalamo-pituitary-adrenal axis · Negative feedback

\begin{abstract}
Objective: The amygdala (AMG) plays a facilitatory role in the hypothalamic-pituitary-adrenal (HPA) axis. The effect of the AMG on the negative feedback exerted by glucocorticoids (GC) is not clear. We investigated the effect of repeated electrical stimulation of the AMG on the feedback action of GC upon the adrenocortical (AC) response to stressful stimuli. Methods: Rats received electrical stimulation into the central amygdalar nucleus once daily for 4 days. At days 5 and 12 after the onset of stimulation, rats were treated with dexamethasone (Dex) or vehicle and were exposed to either photic or acoustic stress stimuli, and serum corticosterone (CS) was measured. In another group of rats, we measured the binding of Dex to the hippocampal cytosol at 5 and 12 days after the AMG stimulation. Results: At 5 and 12 days after the onset of stimulation or a sham control, stress increased the serum CS level. In the sham group, Dex completely inhibited the CS response, but at 5 days after stimulation, it was significantly less effective in doing this. At day 12 , Dex was as effective as in the control group. AMG stimulation delayed the return of CS response to basal levels and caused a significant decrease in the binding capacity of Dex to hip-
\end{abstract}

pocampal cytosol. Conclusion: Electrical stimulation of the AMG caused a transient impairment of the feedback action of GC upon the stress response. This effect may be due to the decrease in hippocampal corticosteroid receptors. This suggests that the impaired GC feedback caused by AMG stimulation may be involved in the facilitatory effect of the AMG on the function of the $A C$ axis.

(c) 2015 S. Karger AG, Basel

\section{Introduction}

It is now well established that there is a bidirectional communication between the nervous system and the immune system. A large body of evidence has confirmed that reciprocal pathways of interaction between these two systems involve a neural pathway which includes direct contact between nerve terminals and lymphoid organs and humoral pathways via circulating glucocorticoids (GC) secreted following activation of the hypothalamopituitary-adrenal (HPA) axis. The activity of the HPA axis is regulated by extrahypothalamic structures including the amygdala (AMG), which plays a significant facilitatory role $[1,2]$. Thus, acute electrical stimulation of the AMG in a variety of animals results in activation of the HPA axis [2]. This effect involves neural mechanisms mediated by amygdalar and hypothalamic norepinephrine 
and serotonin and by the secretion of hypothalamic corticotropin-releasing hormone (CRH-41) [3]. Bilateral lesions of the central and medial AMG have been found to inhibit HPA axis responses following a variety of stress modalities including olfactory, somatosensory, photic and acoustic stimulation, conditioned fear, laparotomy and immobilization stress [4-6]. In addition, several studies show that lesions of the central AMG inhibit the HPA axis responses to immune challenges such as herpes simplex virus infection and systemic injection of IL- $1 \beta$ [7]. We have previously reported that the AMG may facilitate the adrenocortical (AC) response to glutamate injected into the paraventricular nucleus [8].

The activity of the HPA axis is also regulated by the negative feedback exerted by corticosteroids which act predominantly at the hippocampus [9]. The effect of these hormones is mediated by 2 types of intracellular corticosteroid receptors [9-12]. It was previously reported that repeated electrical stimulation of the AMG, resulting in kindling, caused a transient decrease in hippocampal GC receptor (GR) mRNA [13-15], and that this effect was associated with increased fearful behavior [15]. Many studies show that alterations in hippocampal GR activity may affect the responses of the HPA axis due to the impaired feedback action of the GC [16-18].

In view of these observations, we attempted to examine the effect of repeated electrical stimulation of the AMG on the responses to stress stimuli and on the function of the negative feedback exerted by GC.

\section{Materials and Methods}

Male rats (Hebrew University strain) weighing 180-200 g were purchased from Harlan (Hebrew University, Jerusalem) and housed in the animal care facility in compliance with the standard guidelines for animal care. All experiments were approved by the institutional committee for animal care. The rats were given free access to food and water. They were transferred to individual cages $24 \mathrm{~h}$ prior to stress exposure. Each experimental group consisted of 6 animals. All experiments were performed between 7 and 11 a.m.

Animals were anesthetized with ketamine-xylazine intraperitoneally (200 and $10 \mathrm{mg} / \mathrm{kg}$ body weight, respectively) and the stimulating electrodes were stereotaxically implanted into the central amygdalar nucleus. Stereotaxic coordinates [7], taking the bregma suture as the zero reference point, were the anterior-posterior plane $(\mathrm{AP})=0$, lateral plane $(\mathrm{L})=4.0$ and height $(\mathrm{H})=-8.5$. The electrodes consisting of concentric bipolar constructions were fixed to the skull with dental cement insulated to within $0.7 \mathrm{~mm}$ of the tip. One week after electrode implantation, electrical stimulation $(0.5 \mathrm{~mA}$ for $1 \mathrm{~ms}$ and 100/s) was performed under anesthesia for 5 min once daily for 4 consecutive days. Sham stimulation consisted of complete anesthesia and connection of the electrodes to a stimulator but with no current being turned on. At 5 and 12 days after the onset of the electrical stimulation, the rats were exposed to stressful stimuli.

Stress Stimuli

Acoustic Stimulus

Animals were exposed to a ringing bell with an intensity of 109 $\mathrm{dB}$ for $4 \mathrm{~min}$.

Photic Stimulus

Animals were exposed, in a dark room, to a photo stimulator emitting flashes at a rate of $4 / \mathrm{s}$ for $4 \mathrm{~min}$. All animals were sacrificed 15 min after the onset of the stimuli and trunk blood was collected for the determination of corticosterone (CS).

\section{Injection of Dexamethasone}

Animals were injected intraperitoneally with either vehicle or Dex (Sigma), $40 \mu \mathrm{g} / \mathrm{kg}$ of body weight, dissolved in saline containing $1 \%$ ethanol; $3.5 \mathrm{~h}$ later, the animals were exposed to either acoustic or photic stress as described above.

\section{Corticosterone Determination}

CS was determined by radioimmunoassay as previously described [19]. The sensitivity of the assay is $0.5 \mu \mathrm{g} / 100 \mathrm{ml}$. All data are presented as a mean \pm SEM.

\section{Binding Assay}

For the corticosteroid-binding assay, the rats underwent bilateral adrenalectomy $24 \mathrm{~h}$ prior to the assay, in order to clear all binding sites from endogenous CS. The receptor-binding assay was performed as previously described [19]. In brief, aliquots of cytosol fraction of dorsal hippocampal tissue ( $300 \mu \mathrm{l}$, equivalent to $1.5 \mathrm{mg}$ protein) were incubated with a saturating concentration of $20 \mathrm{nM}{ }^{3} \mathrm{H}$-Dex $\left(1,2,4{ }^{3} \mathrm{H}-\mathrm{Dex}, 80 \mathrm{Ci} / \mathrm{mmol}\right.$; Amersham Life Science) for $20 \mathrm{~h}$ at $4^{\circ} \mathrm{C}$. Nonspecific binding was determined in parallel incubations containing a 500-fold excess of unlabeled Dex. Following incubation, aliquots were run on Sephadex LH-20 (Sig$\mathrm{ma}$ ) to separate bound and free ligands. Specific binding was expressed as femtomoles of Dex/mg protein.

\section{Statistical Analysis}

Analysis was performed with the Sigma-Stat software package (SPSS, USA). Data are presented as mean \pm SEM. The results were analyzed with analysis of variance followed by the Student Newman-Keuls test.

\section{Results}

Figure 1 shows that the acoustic and photic stress stimuli caused a marked 5-fold increase in serum CS in the sham-stimulated animals when compared to the nonstressed controls. A similar response to both stimuli was found in animals at 5 days following the onset of AMG stimulation. In this group, there was also a slight but significant increase of basal CS levels compared to in the sham-stimulated group. In sham-stimulated rats, the administration of 

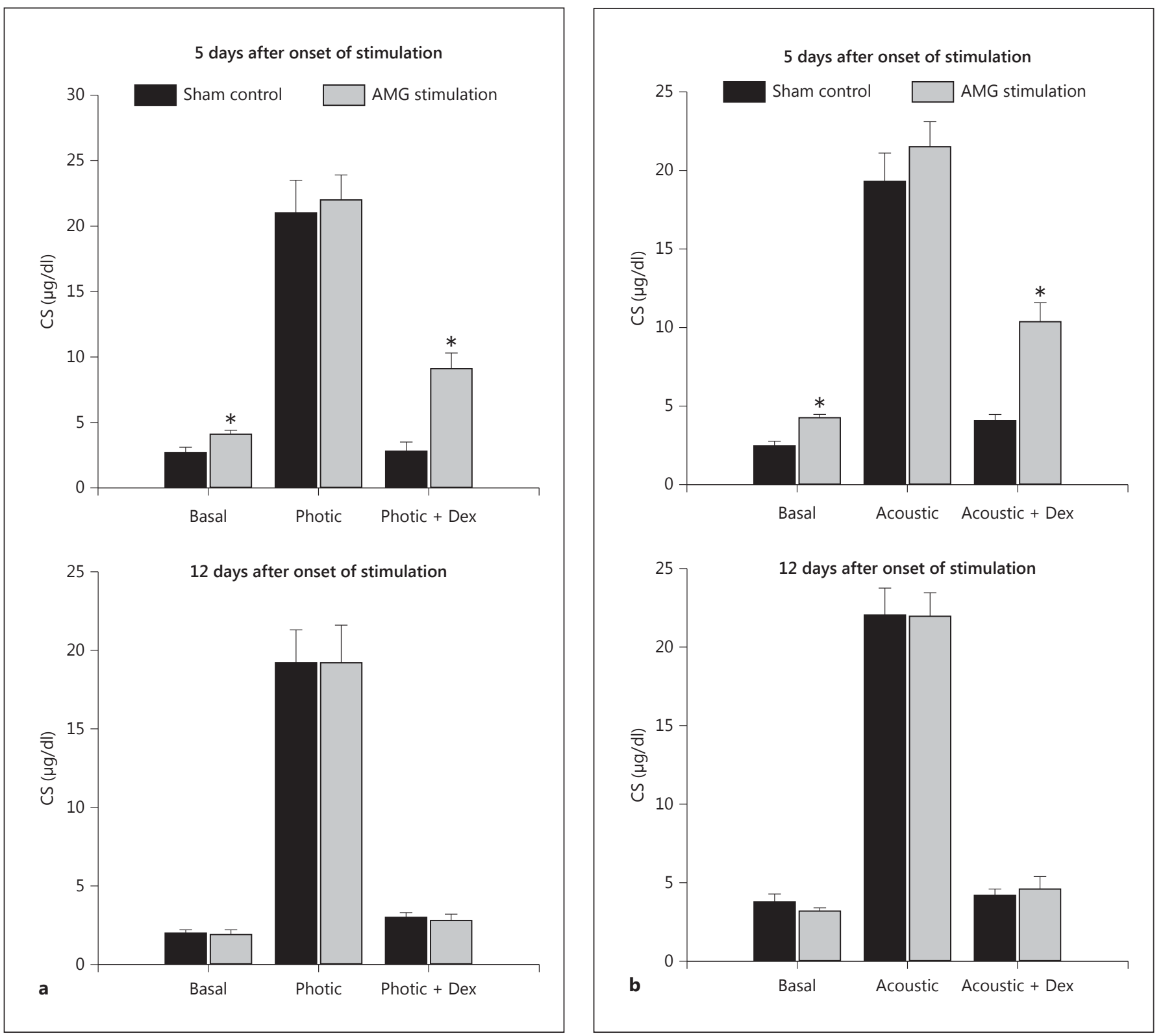

Fig. 1. Effect of AMG electrical stimulation (gray bars) and sham control (black bars) on the adrenocortical response to photic (a) and acoustic (b) stress stimuli in animals treated with vehicle or Dex. Values of serum CS were measured at 5 or 12 days after the onset of stimulation. Each bar represents the mean \pm SEM of CS values. $* \mathrm{p}<0.05$, compared to the respective sham-stimulated group.

Dex completely inhibited the AC responses to both photic and acoustic stimuli. In contrast, 5 days after AMG stimulation, the administration of Dex was significantly less effective in inhibiting the AC response to stress, i.e. Dex inhibited the CS response by only $50 \%$. At 12 days after AMG stimulation, the $\mathrm{AC}$ responses to both stress modalities and the effect of Dex were similar to in sham-stimulated controls.
Next, we examined the effect of AMG stimulation on the ability of endogenous GC to terminate the stress-induced AC response. Figure 2 shows that $30 \mathrm{~min}$ after the onset of acoustic stress, CS serum levels markedly decreased by $60 \%$ (compared to the peak level at $10 \mathrm{~min}$ ). However, at 30 min after stress stimulation in the AMGstimulated group, serum levels of CS were significantly 


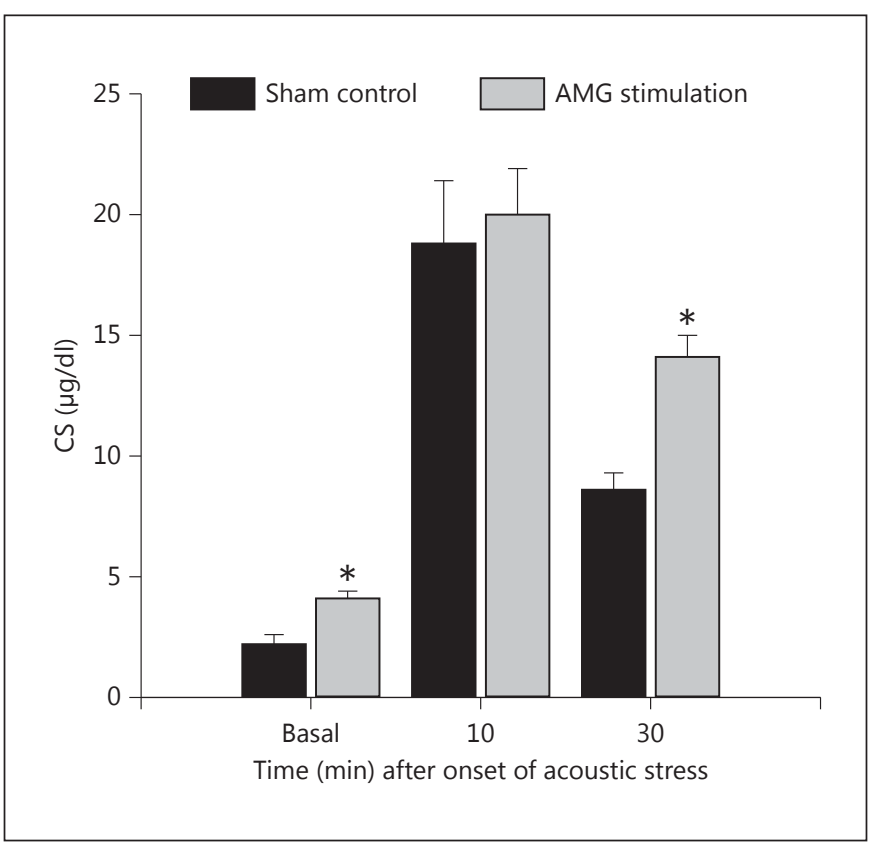

Fig. 2. Effect of AMG electrical stimulation (gray bars) and sham control (black bars) on adrenocortical response to acoustic stress. The animals were exposed to acoustic stress 5 days following the onset of AMG stimulation, and serum CS levels were measured at 10 and 30 min following acoustic stress. Each bar represents the mean \pm SEM of CS values. ${ }^{*} \mathrm{p}<0.05$, compared to the sham-stimulated control group.

higher (2-fold) compared to the sham-stimulated control group at this time point.

Finally, we tested if the reduced feedback effect of GC following AMG stimulation is associated with changes in GR hippocampal binding activity. Figure 3 shows that at 5 days after the onset of AMG stimulation, the specific binding of ${ }^{3} \mathrm{H}$-Dex to corticosteroid receptors in hippocampal cytosol was significantly lower (40\%) compared to in the sham-stimulated group. At 12 days after stimulation the binding of Dex returned to normal values, i.e. like in the sham-stimulated group.

\section{Discussion}

The activation of the HPA axis depends on the secretion of CRH-41 from neurons located in the hypothalamic paraventricular nucleus $[1,20]$. In response to a variety of stimuli including immune mediators such as IL-1, CRH-41 is released into the portal system, causing the secretion of pituitary adrenocorticotropic hormone and, consequently, AC GC. The activation of the HPA

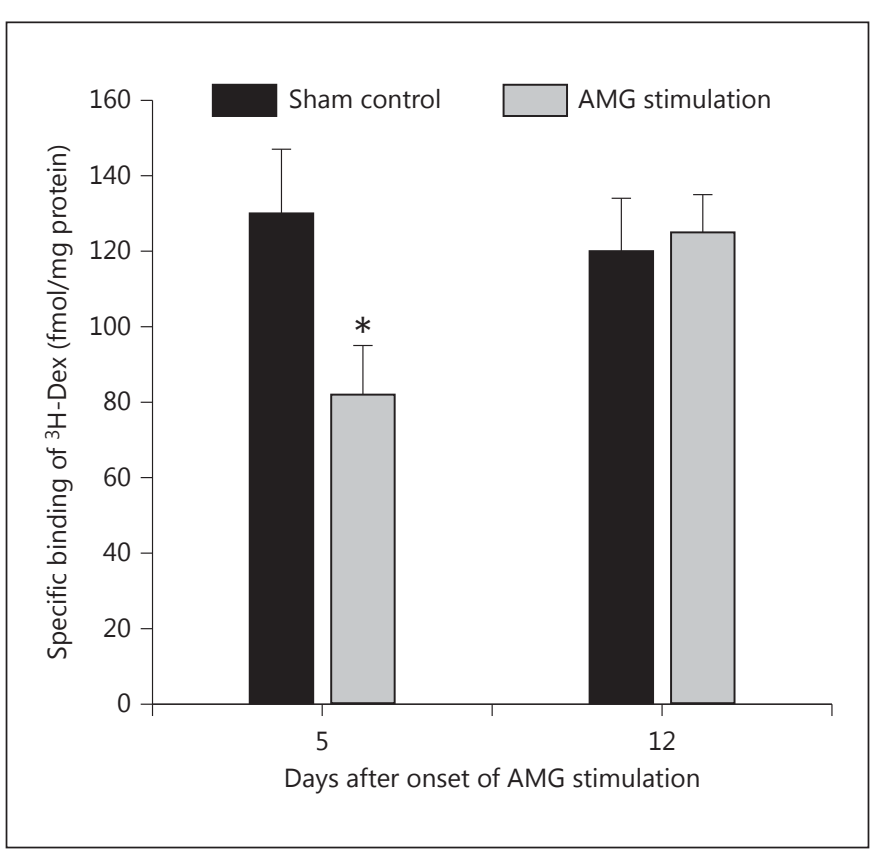

Fig. 3. Effect of AMG electrical stimulation (gray bars) and sham control (black bars) on the binding capacity of ${ }^{3} \mathrm{H}$-Dex by hippocampal cytosol fraction. Hippocampal tissues were incubated with saturating concentration of ${ }^{3} \mathrm{H}-\operatorname{Dex}(20 \mathrm{nM})$ with and without excess of nonradioactive Dex. Each bar represents the mean \pm SEM of 6 hippocampal tissues done in triplicates. ${ }^{*} \mathrm{p}<0.05$, compared to the sham-stimulated control group.

axis is regulated by extrahypothalamic limbic structures which play a significant facilitatory role $[2,6]$. It is well known that the hippocampus plays a major role in the negative feedback action of GC upon the HPA axis $[1,9]$. This effect is mediated by 2 types of corticosteroid receptors, the mineralocorticoid (MR) and the GR. These receptors differ in their localization and their affinities to GR and MR [10, 11, 21]. The relative involvement of each receptor type may depend on the type of stress modality $[2,10,12]$. Neuroendocrine studies show that the AMG projects to several hippocampal areas including the CA1 region $[22,23]$. These neural pathways may potentially affect various aspects of hippocampal functions. However, the influence of the AMG on hippocampal functions related to the regulation of the HPA axis is not yet clear.

We found here that repeated electrical stimulations of the central AMG significantly attenuated the inhibitory action of Dex on the AC responses to both acoustic and photic stress stimuli. In addition, we tested the effect of AMG stimulation on the ability of endogenous GC to terminate the AC stress response. We found that AMG stimulation attenuated the decline in serum CS to basal levels. 
Also, AMG stimulation caused a slight increase in basal levels of CS; this may result from impaired feedback exerted by GC. It should be noted that the effect of AMG stimulation on the AC function is temporary; at day 12 after the stimulations, the AC responses to stress returned to normal values like those in the sham-stimulated groups.

Although we found that the AMG stimulation impaired the negative feedback action of GC, no change was found in the magnitude of the AC response to either acoustic or photic stimuli compared to the control groups. The reason for this apparent discrepancy is not clear.

Previous studies, however, showed that kindling caused by repeated electrical stimulations of the AMG increased the level of MR mRNA in the dentate gyrus of the hippocampus while the mRNA of GR decreased [14]. It is possible that the increase in hippocampal MR, known to modulate the AC responses to stress, may partially balance the feedback effect of circulating GC and thus blunt the effect of AMG stimulation on reducing the negative feedback action of GC.

To examine whether the impaired feedback caused by AMG stimulation may result from decreased hippocampal GR, we measured the binding of ${ }^{3} \mathrm{H}-$ Dex by hippocampal tissue. It has been previously demonstrated that impaired GC feedback is associated with reduced hippocampal corticosteroid receptors. This reduction markedly attenuated the inhibitory effect of the hippocampus over the hypothalamus and impaired the negative feedback effect of GC on the AC responses to various stressful stimuli [17-19].

Our results showed that AMG stimulation caused a significant reduction in the maximal binding capacity of ${ }^{3} \mathrm{H}$-Dex by hippocampal cytosol. It may be assumed, therefore, that the impaired feedback of GC induced by AMG stimulation is mediated by the reduced level of hippocampal corticosteroid receptors.

In agreement with our results, previous studies showed that kindling in rats, induced by repeated AMG stimulations, decreased GC mRNA expression in hippocampal regions [13-15]. The mechanisms by which AMG stimulation caused the reduction of hippocampal GR is not yet clear. It was suggested that this effect is not due to the AMG-induced loss of hippocampal neurons because kindling induced by intense AMG stimulation increases benzodiazepine receptors in the hippocampus [15]. One mechanism which may be involved in reduced hippocampal GR is the downregulation caused by the hypersecretion of GC during AMG stimulations. It is well established that chronic stimulation of GR with GC results in decreased levels of GC binding and GR mRNA [14]. Another mechanism which may be involved in GR downregulation may be the stimulation of hippocampal Nmethyl-D-aspartate receptors causing a rise in the intracellular calcium ion concentration and a downregulation in GR mRNA expression [13].

In conclusion, we showed here that impaired GC feedback induced by repeated AMG electrical stimulations may be involved in the regulatory role of this limbic structure on the HPA axis.

\section{Acknowledgements}

This work was supported by the Lena P. Harvey Endowment Fund for Neurological Research. This article is dedicated to the memory of Dr. Liora Yaar from whom I (J.W.) learned what stress is and how to cope with it.

\section{References}

1 Antoni FA: Hypothalamic control of adrenocorticotropin secretion: advances since the discovery of 41-residue corticotropin-releasing factor. Endocr Rev 1986;7:351-378.

2 Feldman S, Conforti N, Weidenfeld J: Limbic pathways and hypothalamic neurotransmitters mediating adrenocortical responses to neural stimuli. Neurosci Biobehav Rev 1995 19:235-240.

3 Feldman S, Weidenfeld J: The excitatory effects of the amygdala on hypothalamo-pituitary-adrenocortical responses are mediated by hypothalamic norepinephrine, serotonin, and CRF-41. Brain Res Bull 1998;45:389-393.
4 Beaulieu S, Di Paolo T, Cote J, Barden N: Participation of the central amygdaloid nucleus in the response of adrenocorticotropin secretion to immobilization stress: opposing roles of the noradrenergic and dopaminergic systems. Neuroendocrinology 1987;45:37-46.

5 Feldman S, Conforti N: Amygdalectomy inhibits adrenocortical responses to somatosensory and olfactory stimulation. Neuroendocrinology 1981;32:330-334.

6 Gray TS: Amygdaloid CRF pathways. Role in autonomic, neuroendocrine, and behavioral responses to stress. Ann NY Acad Sci 1993; 697:53-60.
7 Weidenfeld J, Itzik A, Goshen I, Yirmiya R, Ben-Hur T: Role of the central amygdala in modulating the pituitary-adrenocortical and clinical responses in experimental herpes simplex virus-1 encephalitis. Neuroendocrinology 2005;81:267-272.

-8 Weidenfeld J, Newman ME, Itzik A, Feldman S: Adrenocortical axis responses to adrenergic and glutamate stimulation are regulated by the amygdala. Neuroreport $2005 ; 16: 1245-$ 1249.

9 Feldman S, Weidenfeld J: Neural mechanisms involved in the corticosteroid feedback effects on the hypothalamo-pituitary-adrenocortical axis. Prog Neurobiol 1995;45:129-141. 
10 de Kloet ER, Oitzl MS, Joels M: Functional implications of brain corticosteroid receptor diversity. Cell Mol Neurobiol 1993;13:433455.

-11 De Kloet ER, Vreugdenhil E, Oitzl MS, Joels M: Brain corticosteroid receptor balance in health and disease. Endocr Rev 1998;19:269301.

$\checkmark 12$ Herman JP: Regulation of adrenocorticosteroid receptor mRNA expression in the central nervous system. Cell Mol Neurobiol 1993;13: 349-372.

13 Bao GS, Cheng XQ, Hua Y, Wang ZD, Liu ZG: Changes of glucocorticoid receptor mRNA expression in basolateral amygdale-kindled rats. Chin Med J 2011;124:2622-2627.

14 Clark M, Smith MA, Weiss SR, Post RM: Modulation of hippocampal glucocorticoid and mineralocorticoid receptor mRNA expression by amygdaloid kindling. Neuroendocrinology 1994;59:451-456.
15 Kalynchuk LE, Meaney MJ: Amygdala kindling increases fear responses and decreases glucocorticoid receptor mRNA expression in hippocampal regions. Prog Neuropsychopharmacol Biol Psychiatry 2003;27:12251234.

16 Jacobson L, Sapolsky R: The role of the hippocampus in feedback regulation of the hypothalamic-pituitary-adrenocortical axis. Endocr Rev 1991;12:118-134.

17 Sapolsky RM, Krey LC, McEwen BS: Glucocorticoid-sensitive hippocampal neurons are involved in terminating the adrenocortical stress response. Proc Natl Acad Sci USA 1984; 81:6174-6177.

18 Schobitz B, Sutanto W, Carey MP, Holsboer F, de Kloet ER: Endotoxin and interleukin 1 decrease the affinity of hippocampal mineralocorticoid (type I) receptor in parallel to activation of the hypothalamic-pituitary-adrenal axis. Neuroendocrinology 1994;60:124133.

19 Bener D, Wohlman A, Itzik A, Yirmiya R, Ben-Hur T, Weidenfeld J: Glucocorticoid resistance following herpes simplex-1 infection: role of hippocampal glucocorticoid receptors. Neuroendocrinology 2007;85:207-215.
20 Plotsky PM, Cunningham ET Jr, Widmaier EP: Catecholaminergic modulation of corticotropin-releasing factor and adrenocorticotropin secretion. Endocr Rev 1989;10:437458.

21 Ratka A, Sutanto W, Bloemers M, de Kloet ER: On the role of brain mineralocorticoid (type I) and glucocorticoid (type II) receptors in neuroendocrine regulation. Neuroendocrinology 1989;50:117-123.

22 Aggleton JP: A description of the amygdalohippocampal interconnections in the macaque monkey. Exp Brain Res 1986;64:515526.

23 Krettek JE, Price JL: Projections from the amygdaloid complex and adjacent olfactory structures to the entorhinal cortex and to the subiculum in the rat and cat. J Comp Neurol 1977;172:723-752. 